\title{
Review of improvements in wire electrode properties for longer working time and utilization in wire EDM machining
}

\author{
Ibrahem Maher • Ahmed A. D. Sarhan • M. Hamdi
}

\begin{abstract}
Wire electrical discharge machining (WEDM) is an important technology, which demands high-speed cutting and high-precision machining to realize productivity and improved accuracy for manufacturing hard materials. WEDM has experienced explosive growth and complexity of equipment as well as rising demand for the basic process tool (the wire electrode). Greater taper angles, thicker workpieces, automatic wire threading, and long periods of unattended operation make the selection of the ideal wire a much more critical basis for achieving successful operation. This paper focuses on the evolution of EDM wire electrode technologies from using copper to the widely employed brass wire electrodes and from brass wire electrodes to the latest coated wire electrodes. Wire electrodes have been developed to help user demand and needs through maximum productivity and quantity by choosing the best wire. In the final part of the paper, the possible trends for future WEDM electrode research are discussed.
\end{abstract}

Keywords WEDM · Coated wires · Brass wires · Steel wires · Diffusion annealed $\cdot$ Composite wire electrodes

\footnotetext{
I. Maher · A. A. D. Sarhan $(\triangle) \cdot$ M. Hamdi

Centre of Advanced Manufacturing and Material Processing, Department of Mechanical Engineering, Faculty of Engineering, University of Malaya, 50603 Kuala Lumpur, Malaysia e-mail: ah_sarhan@yahoo.com

I. Maher

Department of Mechanical Engineering, Faculty of Engineering, Kafrelsheikh University, Kafrelsheikh 33516, Egypt

A. A. D. Sarhan

Department of Mechanical Engineering, Faculty of Engineering, Assiut University, Assiut 71516, Egypt
}

\section{Introduction}

Wire electrical discharge machining (WEDM) is among the more widely known and applied non-traditional machining processes in industry today. In this procedure, improvements to the process mechanism and control have rapidly been taking place. WEDM can machine harder, they are higher strength, corrosive and wear-resistant, and difficult-tomachine materials. With WEDM, it is also possible to machine complicated shapes that cannot otherwise be achieved using traditional machining processes, such as turning, milling, and grinding. Applications of WEDM include extrusion dies, fuel injector nozzles, aircraft engine turbine blades, and machining of difficult-to-machine materials like tool steel, titanium, metal matrix composites (MMCs), and cemented carbides $[1-5]$. Besides machining electrically conductive workpieces, some WEDM work has also been reported on insulating ceramics and non-conductive materials [6-9].

The Russian Lazarenko couple designed the first electrodischarge machine in 1955 [10]. Ten years later, a numerically controllable wire discharge machine was developed, and in 1969, a machine for mass production was built. Most wire discharge machine controllers have been enhanced with computer numerical control equipment. As for wire electrodes, pure copper wires were used in the early 1970 s but at the cost of accuracy and strength. In the second half of the 1970s, brass wire started to be used instead of pure copper wire. In 1980, copper wire electrodes coated with zinc and, in the following year, brass wire electrodes coated with zinc were developed and utilized. Brass wire electrodes with added aluminum or chromium were made as well. From 1990 onwards, brass wire electrodes coated with zinc for highprecision cutting and coated $\mathrm{Cu}-50 \mathrm{mass} \% \mathrm{Zn}$ for high-speed cutting were developed. Subsequently, core materials of stainless wire coated with copper were made and utilized. Also, new types of wire were developed which offered higher 
cutting speed due to increased zinc concentration and wire electrodes made of brass with added titanium and aluminum for improved heat resistance when cutting thick materials [11, 12]. Moreover, other special wire electrodes were developed to meet specific cutting conditions and materials, such as steel, tungsten, molybdenum, and abrasive-assisted and porous wire electrodes [13].

As for WEDM, demand is on the rise for high-speed cutting and high-precision machining for the purpose of improving the productivity of molds as well as for achieving high-quality machined workpieces. Wires used in WEDM are the core of the system. Brass wire electrodes are extensively used as WEDM tools. However, along with recent variations in manufacturing field applications, there is an expanding demand for wire electrodes with superior performance to the conventional brass wire electrodes. High-performance wires, including coated, composite, and diffusion-annealed wires are characterized by high conductivity and good sparking ability. These electrodes are generally zinc-coated wires with a copper-brass alloy or steel core, the brass containing either a small amount of chromium or high concentration of zinc. At present, WEDM users are interested in shortening the machining time of products [14-17]. A new, high-performance EDM wire would be expected to provide both high cutting speed and improved accuracy. Thus, this paper focuses on studying improvements of physical, mechanical, and electrical properties of wire electrodes for high-performance WEDM processes.

\section{Wire EDM process}

In WEDM, material removal is based upon the electrodischarge erosion effect of electric sparks occurring between the wire electrode and workpiece. The two are separated by a dielectric fluid, as shown in Fig. 1. A pulse voltage

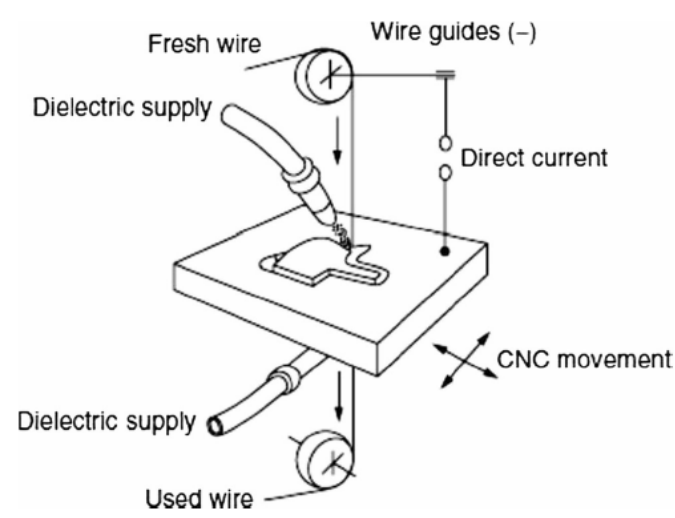

Fig. 1 Wire electric discharge machining (WEDM) schematic diagram is applied between the wire electrode and workpiece in the processing fluid to melt the workpiece surface with the thermal energy of a spark discharge, while simultaneously removing machining dust through a vaporizing explosion and recirculation of the processing fluid. Continuous machining thus becomes possible while running the wire electrode. The residue ensuing from the melting and vaporization of a small volume of the surfaces of both workpiece and EDM wire electrode is contained in a gaseous envelope (plasma). The plasma eventually collapses during off-time. The liquid and vapor phases created by the melting and vaporization of the material are quenched by the dielectric fluid to form solid debris. This process is repeated at nanosecond intervals (depending on the cycle time) along the length of the wire in the cutting zone [18-20].

To achieve a successful operation, selecting the correct wire electrode for WEDM is a very challenging task [21]. As a result, experimentation with different wire electrodes is essential if optimum results are to be achieved. The wire electrodes used in WEDM must have two important characteristics: high electrical conductivity and sufficient mechanical strength. WEDM performance is attributed to mainly six factors, as shown in Fig. 2 [22-24].

\section{Wire electrode properties}

In general, the cutting performance of the WEDM procedure depends on a combination of electrical, mechanical, physical, and geometrical properties of the wire electrode. The factors not related to the wire but which are involved in WEDM, including the mechanical machine concept, improved machine intelligence, use of new pulse generators, and dielectric flushing techniques, also affect machining performance. The following section describes the key physical properties of EDM wires and how they relate to real-world cutting [25-27].

Conductivity is an important property of the EDM wire since it determines how the power supply energy is transferred over the distance from the power feed source to the actual point of cutting. This distance can be considerable, especially if the job is to cut with open guides to clear a workpiece obstruction. Low wire conductivity will result in a voltage drop and associated energy loss over the distance from the power feed to the cutting point. This is not insignificant considering that the peak current of most modern power supplies often exceeds $100 \mathrm{amps}$. Conductivity is often expressed as a percentage of IACS (International Annealed Copper Standard), which is a unit of electrical conductivity for metals and alloys relative to standard annealed copper [28].

Tensile strength indicates the wire's ability to withstand the tension imposed upon it during cutting in order to 
Fig. 2 Effect of various factors improving the WEDM performance [22]

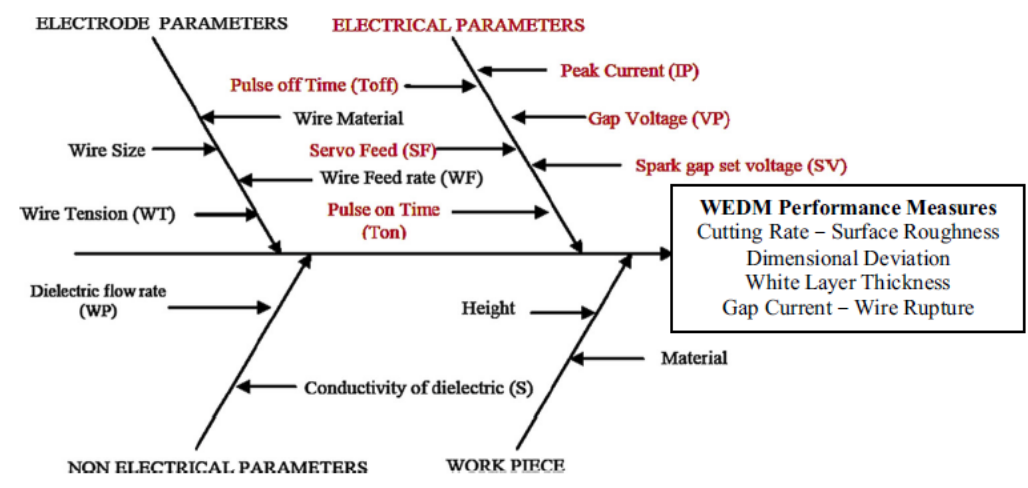

from 0.02 to $0.36 \mathrm{~mm}$ are generally available for WEDM. An increase in diameter results in an increase of pulse energy supplied to the working gap, thus increasing the overall material removal rate $[31,32]$. On the other hand, for micro-WEDM where small pulse energies are predominant, using ultra-fine wires less than 30 microns in diameter is required [33]. A number of other wire electrode shapes have also been conceived in many patents [34-36]. Meanwhile, the use of modified cylindrical wires is rather limited due to their high manufacturing cost. A cutting rate increase of up to $15-20 \%$ has been observed owing to the enhanced heat transfer in six-lobed wire electrodes [37]. Wire electrodes with twisted groves have been patented in order to avoid the occurrence of sparks at the same point [38]. Regarding their patents, several inventors have disclosed that sparks generated in WEDM moved close to the parts and hot spots on the wire electrode are prevented owing to the change in direction of the wire's rectangular cross section [39, 40]. Figure 3 depicts some of the different wire shapes patented to enhance material removal rate $[37,38,40$, 41]. In 1969 , a $0.15-\mathrm{mm}$ diameter wire was applied to achieve the maximum possible cutting rate; the diameter was subsequently enhanced to $0.36 \mathrm{~mm}$. Figure 4 illustrates the increase in wire diameter with the developed wire electrode for WEDM process improvement $[42,11]$. Efforts have been made in the past to identify and analyze the important force components acting on the smaller-diameter wire electrode $(0.03 \mathrm{~mm})$, like electrostatic, electromagnetic, dielectric flushing, wire traction, wire feed, etc. [43, 44].

Like most other things in life, finding the optimum wire for any application means reaching an acceptable compromise among the above-mentioned properties, since they are frequently conflicting. For example, high conductivity wires often have low tensile strength. 




Fig. 3 Customized wire shapes [37, 38, 40, 41]

\section{Development of EDM wire electrodes}

The first machines in the early 70 s were extremely slow, with cutting rates of about $21 \mathrm{~mm}^{2} / \mathrm{min}$. Cutting rates went up in the early $80 \mathrm{~s}$ to $64 \mathrm{~mm}^{2} / \mathrm{min}$, while today, machines are equipped with automatic wire threading and can cut over 20 times faster than the earliest machines [45, 20].

For high-speed cutting and high-precision machining, any wire electrode should have key physical properties, viz high electrical conductivity, tensile strength, elongation, melting point, and straightness [46-48].

Technologies with brass-coated wires are categorized as HIH (high hawk), HIF (high falcon), HIE (high eagle), HIR (high real), and HIS (high sonic) [27]. HIS and HIR wire electrodes were developed for high-speed EDM applicability and are being utilized for mass production applications like metal molds for IC lead frames and electronic parts. Figure 5

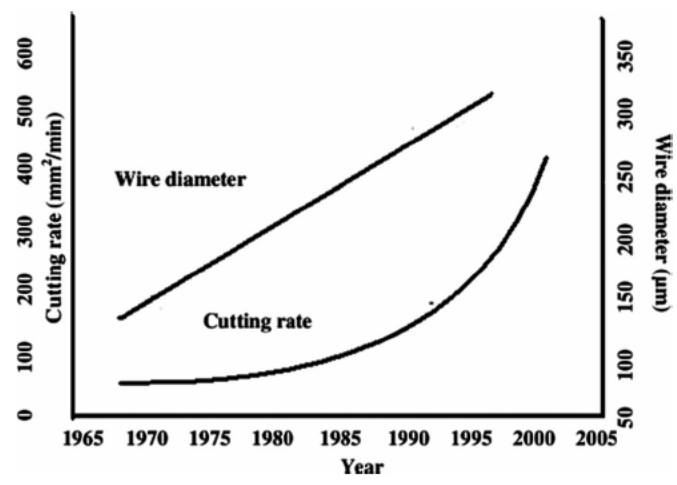

Fig. 4 Higher cutting rate machines using thick wires [11] shows various wire electrodes built for better roughness and flatness with faster cutting speed [49].

Adding zinc to the wire electrode helps control electrical discharge properties, subsequently enhancing machining performance. The addition of conductive alloying elements to the core surface of wire electrodes controls clarification and heat release [50]. Several inventors [51-53] have focused their efforts toward enhancing wire electrode performance by controlling the above methods. Significant increase in the performance of WEDM has been reported with single (plain wire electrodes) or multi-component (zinc-coated, diffusion annealed, etc.) wire electrodes [54, 55]. Brass wire electrodes are extensively used on account of their ability to generate stable discharge, but their electrical conductivity is low. Many wire electrodes have been developed by considering the electrical conductivity and heat resistance of the coated layer or core material. One property that noticeably affects WEDM performance is fracture toughness. Fracture toughness is the ability of the wire electrode to resist breakage and withstand the formation of craters on its surface [56-58]. Wires with high tensile strength can be made, but as a result have a tendency to break. Composite wire electrodes with a steel core and high fracture toughness have been developed to address the wire fracture and electrical conductivity problems. An EDM wire will break when a discharge introduces a flaw into the wire that is greater than the critical flaw size needed to trigger catastrophic failure under the preload tension applied (Fig. 6) [59]. Many high-strength materials, including EDM wires, are notorious for their low fracture toughness, that is, their inability to withstand relatively small flaws without failing. Each and every discharge in the WEDM process makes a crater, which is termed a defect or flaw, in both the wire and workpiece. As flushing conditions deteriorate, those flaws tend to become larger and larger, eventually causing disastrous wire failure [60-62]. Figure 7 shows the rapid advancement rate of WEDM with standard wire and highperformance wire electrodes since its introduction [42, 63, 64].

\section{Plain EDM wires}

Typically, brass wire begins as a continuously cast $20-\mathrm{mm}$ diameter rod. This rod is either cold rolled or cold drawn until it is approximately a 6-mm round or hexagonal cross section. The wire is then annealed and drawn through a series of dies until it is around $0.9 \mathrm{~mm}$ in diameter. In this state, it is commonly called a "redraw" wire. The redraw wire is subsequently drawn through another series of diamond dies until it reaches the final size. At final size, the wire is resistively annealed or thermally tempered in an inert atmosphere, cleaned, and then spooled [65-67]. 
Fig. 5 Characterization of electrical discharge wire electrode [49]

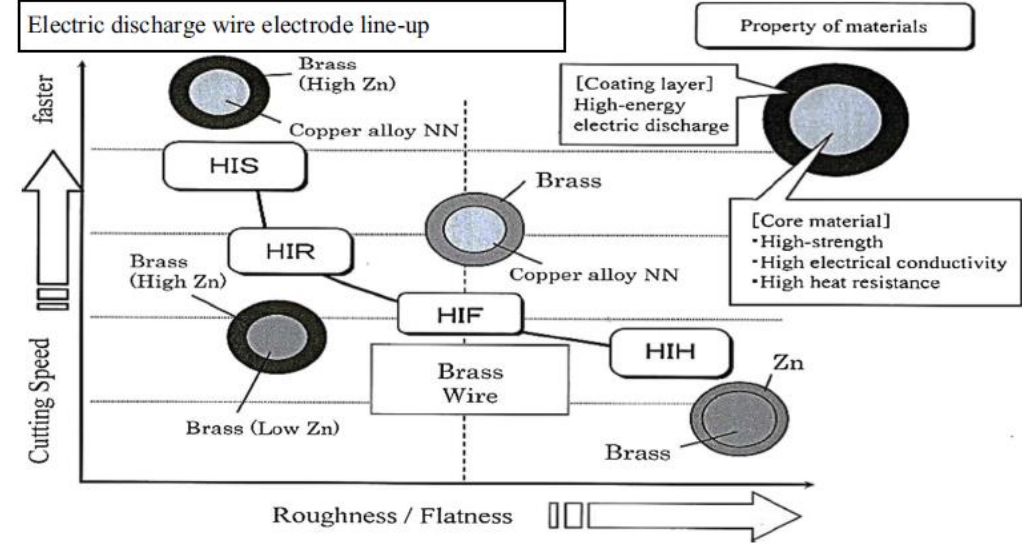

In the EDM wire trade, plain merely means the wire consists of a single homogeneous component and does not have a coated or composite construction [30].

\subsection{Copper wire}

Copper wire, shown in Fig. 8, was the original material used in WEDM. At the time, it was believed that because copper wire had high electrical conductivity it would make the ideal EDM wire. Unfortunately, copper wire has low tensile strength, high melting point, and low vapor pressure rating [68]. This soon became apparent with the development of the secondgeneration pulse-type power supplies, and copper wire was shortly replaced by brass wire. It should be noted that copper wire is still used occasionally for applications in which zinc (contained in brass or coated wires) is considered an unacceptable contaminant $[69,30]$.

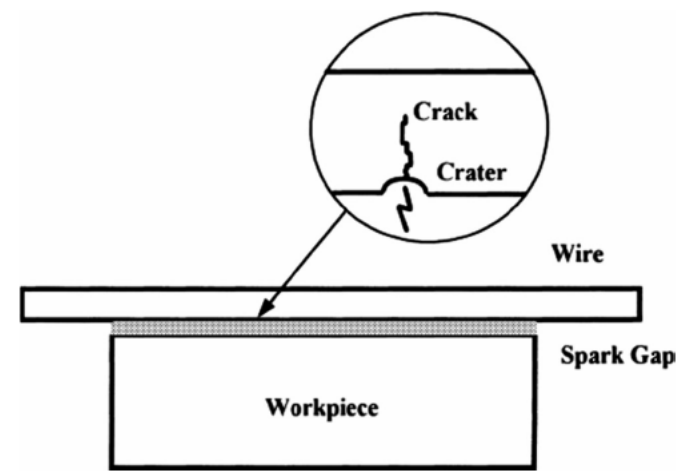

Fig. 6 Showing a defect and a fracture in the wire EDM process [62]

\subsection{Brass wire}

Various attempts have been made in the past to improve wire electrodes. Brass EDM wire is a combination of copper and zinc, typically alloyed in the range of $63-65 \% \mathrm{Cu}$ and $35-$ $37 \% \mathrm{Zn}$ (Fig. 9) [63]. It has been discovered that the addition of zinc improves cutting performance and speed compared to copper in several ways [46]. During the cutting process, the zinc in the brass wire actually boils off or vaporizes, which helps cool the wire and deliver more usable energy to the work zone. Adding zinc provides significantly higher tensile strength and greater vapor pressure rating, which offsets the relative losses in conductivity. Also, some zinc particles that are not filtered out of the dielectric fluid would remain in the gap between the electrode and the workpiece to help the gap ionization and cutting process [42]. Brass rapidly became the most widely used electrode material for general-purpose wire EDM.

It is worth noting that even a small amount of zinc added to copper wire severely reduces conductivity. The conductivity of hard brass wire is typically only $20 \%$ that of copper wire. Since it is zinc that gives the brass wire its improved flushability, some manufacturers now offer a high-zinc brass of $\mathrm{Cu} 60 \% \mathrm{Zn} 40 \%$. This increase in zinc content can increase cutting speed up to $5 \%$ in some optimized applications [70]. However, it is not practical to cold-draw wire with zinc content in excess of $40 \%$. Changes in the wire grain structure make the wire too brittle for further processing into the fine diameters necessary for wire EDM. In some circumstances, significant brass deposit can remain on the workpiece after the cut, which is proven to be difficult to remove. These facts have led to the development of coated wires $[71,72,14]$. 
Fig. 7 Advancement of the WEDM rate since its development

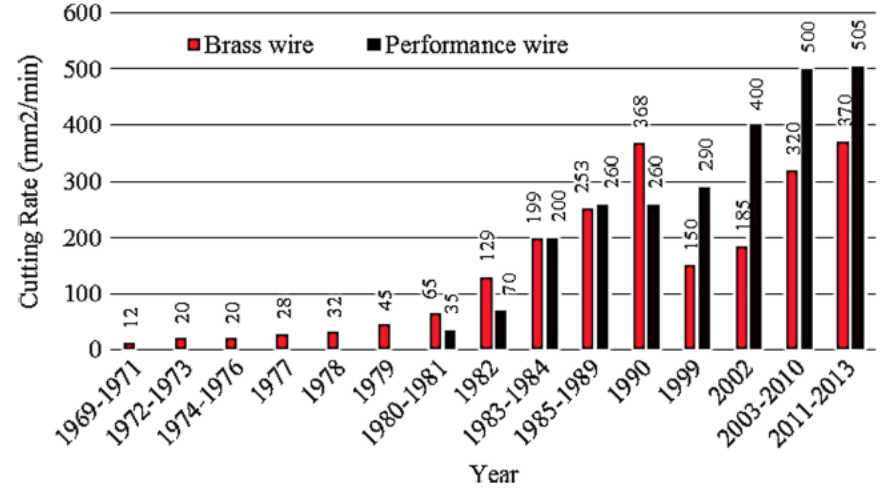

\subsection{Aluminum-brass wire}

Adding a small percentage of aluminum to a brass wire creates a specialty alloy wire, as shown in Fig. 10. Such alloy additions improve the wire's tensile properties, allowing for tensile strength to be brought up to as high as 1,200 MPa without adversely affecting elongation. Some users claim these wires are less prone to breakage than other types of plain brass wire [30].

\section{Coated EDM wires}

US Patent No. US1896613-1933 is one of the earliest patents on zinc-coated wires directed toward improving the quality of such wires [73].

Due to the limitations in producing plain brass wire with alloy percentages greater than $40 \%$ zinc, coated wires were developed in an attempt to put zinc on the surface of the wire while retaining a core wire material that could be successfully drawn [74]. Coated wires are produced by plating or hot dipping redrawn wire and subsequently drawing it to the final size. This is a difficult process since the plated surface zinc has to endure the final drawing process and still present a uniform

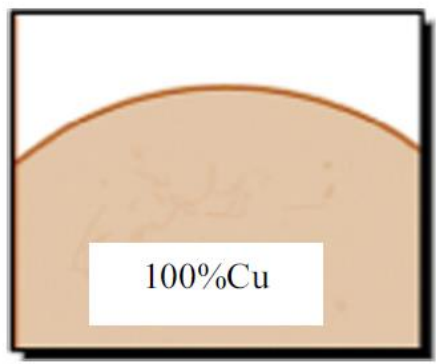

Fig. 8 Solid copper [30] coating to the cut. Currently, no EDM wires are manufactured by a process in which the coating is deposited at the final wire size. They typically have a core of brass or copper for conductivity and tensile strength and are electroplated with a coating of pure or diffused zinc for enhanced spark formation and flush characteristics [75-81].

\subsection{Single-layer coated wires}

Zinc-coated brass wire was one of the first attempts to present more zinc to the wire's cutting surface. This wire consists of a thin (around 5 microns) zinc coating over a core, which is one of the standard EDM brass alloys (Fig. 11). Zinc-coated brass wire offers a significant increase in cutting speed over plain brass wire, with no loss in any of the other critical properties [82]. Exceptional surface finish can be obtained when cutting tungsten carbide, and this wire is often utilized for cutting polycrystalline diamond and graphite. This wire is also utilized in circumstances in which brass wire produces unacceptable brass plating on the workpiece.

Zinc-coated copper wire was another early attempt to combine the conductivity of a copper core with the flushability of zinc. It has no current application because when sparks penetrate the thin zinc coating, the cutting rate slows to the sluggish pace of pure copper wire [83,84].

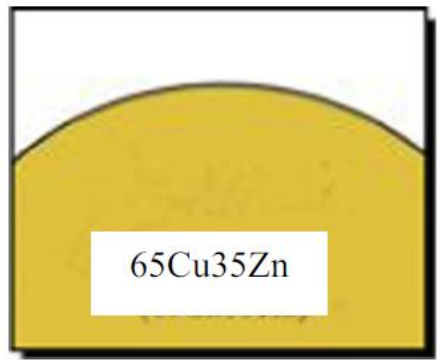

Fig. 9 Solid brass [30] 


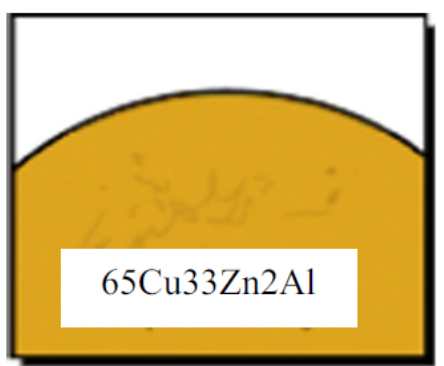

Fig. 10 Aluminum-brass alloy [30]

US Patent No. US4287404 disclosed that coating a wire electrode with a metal or alloy having a low vaporization temperature, such as zinc, cadmium, tin, lead, antimony, bismuth, or alloys thereof, protects the core of the wire against thermal shock resulting from the occurrence of electrical discharge. Additionally, increasing the frequency of the electrical discharges without running the risk of rupturing the wire is feasible [85]. Patent No. US6300587 is for a wire electrode containing a core, and coating layer formed on the outer periphery of the core contains copper. The coating layer comprises an alloy of 55.5 to $75 \mathrm{wt} \%$ copper and at least one element selected from the group consisting of $\mathrm{Zn}, \mathrm{Cs}, \mathrm{Se}$, $\mathrm{Te}$, and Mg. The coating layer does not have an oxide film thereon other than a natural oxide film [86]. Patent No. US6348667 refers to a wire electrode that prevents the corrosion, particularly of the non-eroded surfaces of the hard metal block. This purpose is attained by selecting a wire electrode for the spark-erosive cutting of hard metals, the outer coat of which consists of a metal or metal alloy and which is not nobler than the binder contained in the hard metal. Thus, small metal particles of the outer coat come loose during cutting due to wear of the outer wire electrode coat, and they remain in the dielectric. However, since these small metal particles are not nobler than the metal contained in the hard metal, electrochemical corrosion, namely pitting of the hard metal, can therefore not occur as soon as the small metal particles come

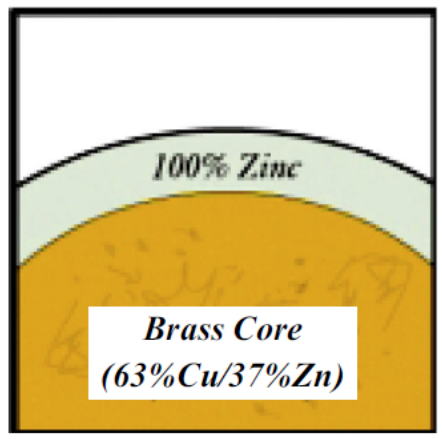

Fig. 11 Zinc-coated brass wire [30] into contact with the hard metal block. Consequently, the pitting phenomena in the hard metal block are avoided. As a rule, the hard metal block contains cobalt, aluminum, magnesium, zinc, or iron [87].

\subsection{Double-layer coated wires}

US Patent No. US4968867 discloses a wire electrode with a core wire having relatively high thermal conductivity, a lower coating layer formed by a low boiling point material and an outermost brass layer with high mechanical strength. Here, the core wire is made of copper, silver, aluminum, or alloys (Fig. 12). Certain effects, such as the vibration dumping effect, heat transfer effect, and resistance to breakage, were observed, all of which ultimately increase machining speed. Figure 12a shows a cross-sectional view of the first embodiment of the wire electrode invented. A core wire (6) is covered by a coating layer (7). In this embodiment, brass containing $35 \%$ $\mathrm{Zn}$ is used for the core wire coating layer, and a $\mathrm{Cu}-\mathrm{Sn}$ alloy including $0.15 \% \mathrm{Sn}$ balanced by $\mathrm{Cu}$ is used for the core wire. Besides brass, a $\mathrm{Cu}$ alloy containing $\mathrm{Cu}$ as the main component and less than $50 \% \mathrm{Mg}$ and/or Cd may be used for the core wire coating layer. As the second embodiment of the present invention, a wire electrode in composite form is obtainable by determining the coating layer thickness so that the cross-sectional area of the coating layer to the entire crosssectional area of the wire electrode of the first embodiment is in the range of 50-90\%. In this embodiment, tensile strength is large, as the coating layer thickness is large too. The crosssectional area of the coating layer to the total cross-sectional area of the wire electrode is about $69 \%$. In the third embodiment, the core wire (6) is covered by a layer (7) which is in turn covered by a layer (8), as shown in Fig. 12b. For the wire electrode, $\mathrm{Zn}$ is used in the outermost layer. The outermost layer is formed by a metallic material including $\mathrm{Zn}, \mathrm{Cd}$, or $\mathrm{Mg}$ as the major component, which has a low boiling point. Accordingly, machining speed can be improved. Figure 12c shows a cross-sectional view of the fourth embodiment. The outermost layer (9) is an oxide film onto coating layer (7). Unnecessary electric discharges at the lateral side of the wire electrode can be reduced because an oxide layer forms on the wire electrode surface. This realizes narrow machined groove width. In the fifth embodiment, the wire electrode constitutes a core wire (6) of a $\mathrm{Cu}$ alloy having high thermal conductivity, a layer (10) that is formed by twisting thin wires of brass with high mechanical strength, and an outermost layer (11) formed by $\mathrm{Zn}$ or $\mathrm{Zn}$ alloy (Fig. 12d). It was confirmed that the electric discharge characteristic can be increased to thereby realize uniform electric discharge so that the surface roughness of a workpiece can be improved. This is because the coating layer, as an intermediate layer with high mechanical strength, is covered by a low boiling point material. Figure $12 \mathrm{e}$ shows a cross-sectional view of the sixth embodiment. The wire

Link to Full-Text Articles:

http://link.springer.com/article/10.1007/s00170-014-6243-3 